Burkard Hillebrands André Thiaville (Eds.)

\title{
Spin Dynamics \\ in Confined \\ Magnetic \\ Structures III
}

With 164 Figures, 42 in Color

Springer 


\section{Contents}

Precessional Switching of Thin Nanomagnets with Uniaxial Anisotropy

Thibaut Devolder, Hans Werner Schumacher, Claude Chappert ...... 1

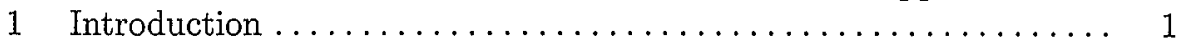

2 Basic Facts About Precessional Dynamics in Transverse Field ..... 3

2.1 From Stoner-Wohlfarth Energy Minimization to Precessional

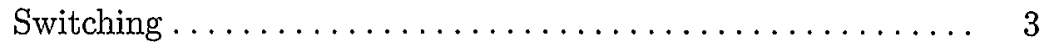

2.2 Definitions: Geometry and Notations .............. 6

2.3 A Tutorial Example: The Bascule Precession of a Isotropic Thin Film ............................ 7

2.3.1 Magnetization Trajectory for a Loss-Free Isotropic Film Subjected to a Transverse Field ........... 7

2.3.2 Switching Speed for a Loss-Free Isotropic Film

Subjected to a Transverse Field $\ldots \ldots \ldots \ldots \ldots \ldots, 9$

3 Precessional Switching at High Hard-Axis Fields $\ldots \ldots \ldots \ldots \ldots, 12$

3.1 "All-Electrical" Experimental Methods .............. 12

3.2 Evidence of the Quasiperiodic Nature of the Magnetization Motion .............................. 13

3.3 Evidence of Quasiballistic Magnetization Switching Trajectories 16

4 Precessional Switching at Moderate Hard-Axis Fields and Zero

Damping .............................. 19

4.1 Classification of the Magnetization Trajectories ......... 20

4.1.1 Field-Dominated (Switching) Trajectories ......... 20

4.1.2 Anisotropy-Dominated (Nonswitching) Trajectories .... 22

4.2 Characteristic Times Involved in a Precessional Switching Event Near and Below $H_{k} \ldots \ldots \ldots \ldots \ldots \ldots \ldots \ldots, 22$

4.2.1 Initial Delay, Slow-Down Time and Maximum Speed Time ............................. 23

4.2.2 Rule of Thumb for the Precession Frequency ........ 24

4.3 Minimal Switching Field, Bifurcation Trajectory and its Intrinsic Noise Sensitivity ................... 26

4.4 Arbitrary Initial Magnetization and Consequences for Nonmacrospin Samples .................... 27

4.5 Minimal Reversal Field Versus Minimal Energy Cost ...... 29

4.6 Perspectives for the Speed Scaling of Precessional MRAM ... 30 
5 Damped Nanomagnets: Relaxation-Dominated Precessional

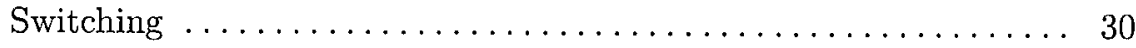

5.1 Consequences of Finite Damping. . . . . . . . . . . . . . 31

5.1 .1 Loss of Energy and of Periodicity ............. 31

5.1 .2 Rise-Time Constraints . . . . . . . . . . . . . 31

5.2 Perturbation Treatment of Finite Damping. . . . . . . . . . . 32

5.2.1 Energy Loss Per Unit Cycling of the Trajectory . . . . . 32

5.2.2 Number of Trajectories Gone Through Before Bifurcation 33

5.3 Bifurcation Field in the Presence of Finite Damping. . . . . . . . 34

6 Direct-Write: Biasing the Precession with an Easy-Axis field . . . . 36

6.1 Precessional Switching in Magnetic Random Access Memories 36

6.2 Magnetization Trajectories for Combined Easy-Axis

and Hard-Axis Fields. . . . . . . . . . . . . . . . . . . . 37

6.2.1 Magnetization Trajectories for Nearly Transverse Applied Fields .................... 38

6.2.2 Magnetization Trajectories for Nearly Antiparallel Applied Fields ...................... 40

6.2.3 General Case: Lobe Centers and Bounce-Occurrence Criteria ............................ 41

6.3 The Dynamical Astroid ..................... 43

6.3.1 Derivation of the Dynamical Astroid ... . . . . . . . 43

6.3.2 Smallest Switching Field and Extrema of the Dynamical Astroid Curve ..................... 43

6.4 Characteristic Duration of a Precessional Direct-Write Event . 45

6.4 .1 Generalized Initial Delay . . . . . . . . . . . . . . . . 45

6.4 .2 Main Rotation Time . . . . . . . . . . . . . . 46

6.4 .3 Alignment Time.................... 46

6.4 .4 Pause Interval $\ldots \ldots \ldots \ldots \ldots \ldots \ldots \ldots \ldots \ldots \ldots$

6.4.5 Precession Frequency for Arbitrary Field Orientation . . 47

6.5 Strategies for the Cell Writing and Selection in a Practical

Memory Array . . . . . . . . . . . . . . . . . . . 49

6.5.1 The Selectivity and Direct-Write Issues

in Precessional MRAM ................ 49

6.5.2 Optimal Field Synchronization for Jitter Immunity . . . 50

6.5.3 Size of the Addressing Window . . . . . . . . . . 50

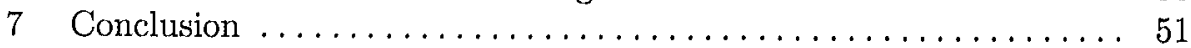

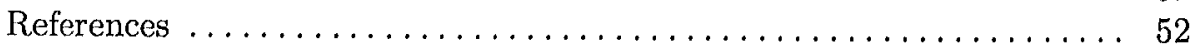

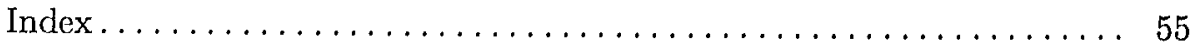

\section{Spin-Wave Excitations}

in Finite Rectangular Elements

Christian Bayer, Jörg Jorzick, Sergej O. Demokritov, Andrei N.

Slavin, Konstantin Y. Guslienko, Dmitry V. Berkov, Natalia L. Gorn, Mikhail P. Kostylev, Burkard Hillebrands . . . . . . . . . . . . . . 57

1 Introduction $\ldots \ldots \ldots \ldots \ldots \ldots \ldots \ldots \ldots \ldots \ldots \ldots \ldots$ 
2 Samples and Experimental Setup .................... 60

3 Experimental Results ........................... 62

3.1 Longitudinally Magnetized Long Stripes ............. 62

3.2 Transversely Magnetized Long Stripes ............... 67

3.3 Rectangular Elements . . . . . . . . . . . . . . . . . . . . 72

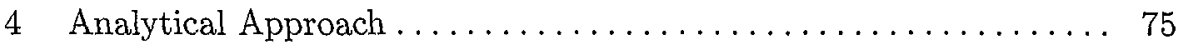

4.1 Longitudinally Magnetized Long Stripes ............. 78

4.2 Transversely Magnetized Long Stripes ................ 81

4.3 Rectangular Magnetic Elements . . . . . . . . . . . . . . 89

5 Micromagnetic Simulations $\ldots \ldots \ldots \ldots \ldots \ldots \ldots \ldots \ldots \ldots . \ldots 3$

6 Outlook ..................................... 98

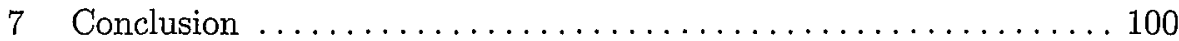

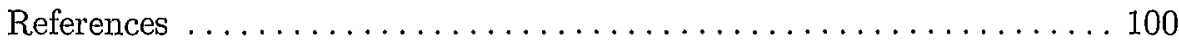

Index .......................................... 103

Ferromagnetic Resonance Force Microscopy

Philip E. Wigen, Michael L. Roukes, Peter C. Hammel ............ 105

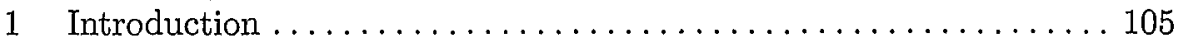

2 MRFM Detection of Weakly Interacting Spins (ESR and NMR) ... 107

3 MRFM in Ferromagnetic Systems . . . . . . . . . . . ....... 109

3.1 Magnetostatic Modes............................ 110

3.2 Linewidths .................................. 112

3.3 Scanning Mode ............................. 114

3.3.1 Dependence of the Fundamental Mode on Sample Dimensions ..................... 116

3.3.2 Dispersion Relation ...................... 120

3.3.3 Spatial Mapping of Magnetostatic Modes ............ 120

3.3.4 Hidden Modes ........................... 121

3.3.5 Mapping RF Force Fields $\ldots \ldots \ldots \ldots \ldots \ldots \ldots \ldots . \ldots \ldots$

3.4 FMRFM in Metal Films ........................ 123

4 Torque Measurements in a Uniform Field ................. 124

4.1 Mechanical Torque on a Thin Film ................. 125

4.2 Magnetization versus Field $(M-H)$ Loops............. 125

4.3 Microresonating Torque Magnetometer $(\mu R T M) \ldots \ldots \ldots \ldots 126$

4.4 Bimaterial Micromechanical Calorimeter Sensor for FMR . . . 128

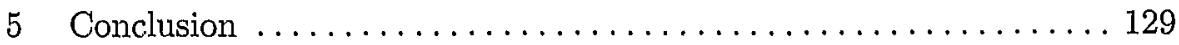

References .................................... 132

Index .......................................... 136

\section{Vortex Dynamics}

Christian H. Back, Danilo Pescia, Matthias Buess $\ldots \ldots \ldots \ldots \ldots 137$

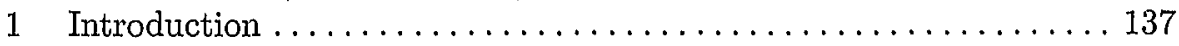

2 Experimental Techniques ......................... 138

2.1 Time-Resolved Magneto-Optical Microscopy ............ 138

2.2 Brillouin Light Scattering ...................... 139 
2.3 Synchrotron-Based Techniques . . . . . . . . . . . . . . 140

$3 \quad$ Excitation Schemes ............................. 141

3.1 Pulsed Precessional Motion ...................... 141

3.2 Microwave Excitation ......................... 142

4 The Energy Landscape of Confined Magnetic Structures......... 142

5 Experimental Results ............................ 143

5.1 Gyroscopic Vortex Motion in Micrometer Sized

Ferromagnetic Squares and Disks ................ 144

5.2 The Modal Spectrum of Permalloy Disks . ............. 147

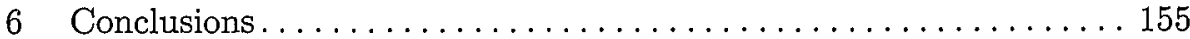

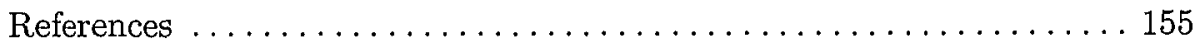

Index ........................................... 160

Domain-Wall Dynamics in Nanowires and Nanostrips

André Thiaville, Yoshinobu Nakatani $\ldots \ldots \ldots \ldots \ldots \ldots \ldots \ldots \ldots \ldots$

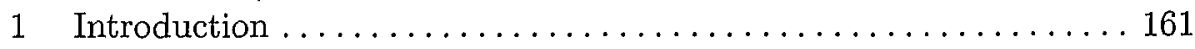

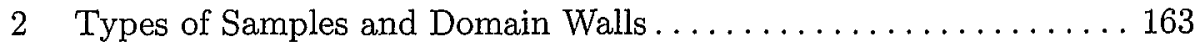

2.1 Wall Types in Nanowires ....................... 164

2.1.1 Numerical Results ........................ 164

2.1.2 Comparison to 1D Profiles................... 169

2.2 One-Dimensional Effective Model $\ldots \ldots \ldots \ldots \ldots \ldots \ldots \ldots 169$

2.3 Wall Types in Nanostrips ...................... 173

3 Dynamics of a Transverse Wall in a Nanowire .............. 174

3.1 Numerical Results . . . . . . . . . . . . . . . . . . . 176

3.2 Analytical Model .......................... 177

4 Dynamics of a Transverse Wall in a Nanostrip ............ 187

5 Dynamics of a Bloch-Point Wall in a Nanowire ................ 191

6 Dynamics of a Vortex Wall in a Nanostrip ................ 195

7 General Results for Steady-State Motion of Domain Walls ....... 198

7.1 Thiele's Equations . . . . . . . . . . . . . . . . . . . . 198

7.2 Döring's Kinetic Potential $\ldots \ldots \ldots \ldots \ldots \ldots \ldots \ldots \ldots \ldots$

8 Conclusions and Outlook .......................... 201

References ..................................... 202

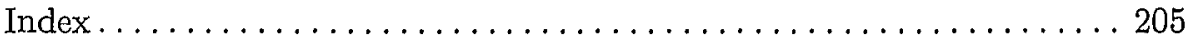

\section{Domain-Wall Dynamics}

in Magnetic Logic Devices

Del Atkinson, Colm C. Faulkner, Dan A. Allwood, Russell P. Cowburn 207

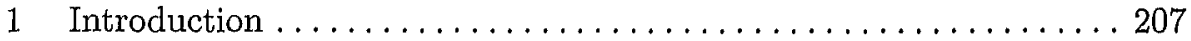

2 Domain-Wall Propagation in Nanowires -Velocity and Dynamics .. 208

2.1 Domain-Wall Dynamics in Continuous and Nanostructured

Permalloy Films ........................... 209

2.2 Domain-Wall Velocity in Permalloy Thin Films ......... 209

2.3 Domain-Wall Behavior in Permalloy Planar Nanowires . . ... 210 
3 Domain-Wall Dynamics in Complex Structures for Magnetic Logic. 214

3.1 Magnetic Logic Circuits ....................... 214

3.2 Domain-Wall Propagation through Corner Structures ...... 216

3.3 Domain-Wall Propagation through Logic Junction Structures. 219

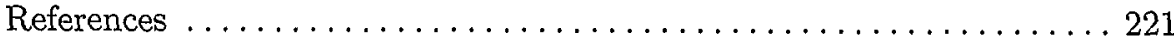

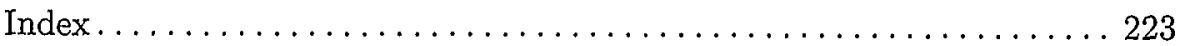

Spin-Transfer Torque and Dynamics

Mark D. Stiles, Jacques Miltat ....................... 225

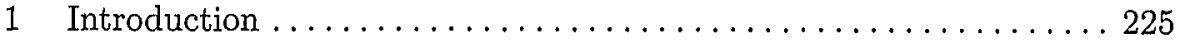

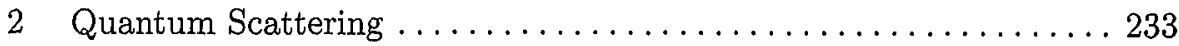

3 Semiclassical Transport ............................ 242

3.1 Boltzmann Equation and Drift-Diffusion Approximation ..... 243

3.2 Spin Pumping .............................. 247

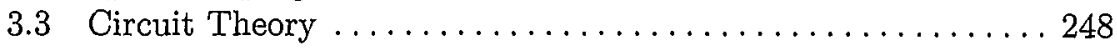

3.4 Collinear Transport .......................... 250

3.5 Non-Collinear Transport and Torque $\ldots \ldots \ldots \ldots \ldots \ldots \ldots 253$

3.6 Leads/Reservoirs ............................ 258

3.7 Lateral Inhomogeneity....................... 258

4 Micromagnetics ................................ 260

4.1 Geometry and Energetics ..................... 260

4.2 Landau-Lifshitz-Gilbert Equation ................. 263

4.3 The Single or Macrospin Approximation .............. 266

4.3.1 Onset of Precessional States ................. 267

4.3.2 Precessional States: Stability Range ............ 269

4.3.3 Switching and Out-of-Plane Precessional States ...... 272

4.4 Langevin Dynamics .......................... 274

4.5 The Micromagnetic Regime $\ldots \ldots \ldots \ldots \ldots \ldots \ldots \ldots \ldots . \ldots \ldots 1$

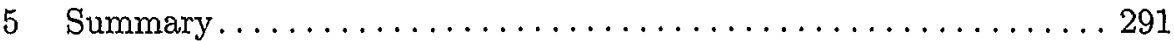

A Appendix Drift-Diffusion Solution for a Single Interface . . . . . . 293

B Appendix Precession and Spin Transfer in Phase Space: Melnikov's

Method ................................... 295

References ..................................... 300

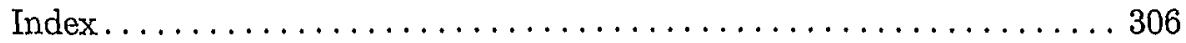

Spin- and Energy Relaxation of Hot Electrons at GaAs

\section{Surfaces}

Torsten Ohms, Kevin Hiebbner, Hans Christian Schneider,

Martin Aeschlimann ............................... 309

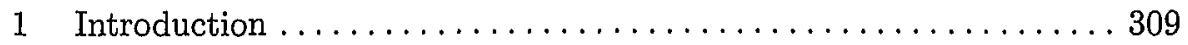

2 Review of Spin-Flip Processes in GaAs . ................. 311

2.1 Optical Orientation of Photoexcited Carriers ............ 311

2.2 Band-Structure Properties . . . . . . . . . . . . . . . . . . . 313

2.3 Elliott-Yafet Mechanism....................... 316

2.4 Dyakonov-Perel Mechanism ..................... 316 
XIV Contents

2.5 Bir-Aronov-Pikus Mechanism . . . . . . . . . . . . . . 317

3 Spin-Polarization Dynamics . . . . . . . . . . . . . . . . 319

3.1 Theory of Spin-Relaxation Dynamics . . . . . . . . . . . . . 319

3.2 Numerical Results . . . . . . . . . . . . . . . . . . . 322

3.3 Spin Decay in a Schottky Barrier .............. 325

4 Experiments . . . . . . . . . . . . . . . . . . . . . . . 327

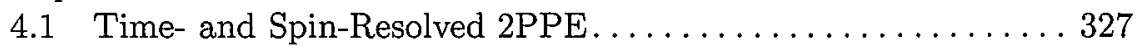

4.2 Experimental Setup . . . . . . . . . . . . . . . . 328

4.3 Experimental Results for GaAs $(100) \ldots \ldots \ldots . \ldots . \ldots . \ldots 330$

4.4 Comparison Between Experimental and Theoretical Results . . 335

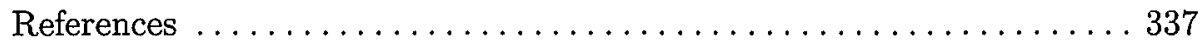

Index . . . . . . . . . . . . . . . . . . . . . . . . . . . . . 340

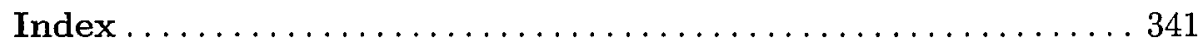

